\title{
Increasing prevalence of antimicrobial resistance among Enterobacteriaceae uropathogens in Bangui, Central African Republic
}

\author{
Raymond Bercion, Dahlia Mossoro-Kpinde, Alexandre Manirakiza, Alain Le Faou
}

Federation of Laboratories, Hôpital Principal, Dakar, Senegal, Institut Pasteur de Bangui, Bangui, Central African Republic

\begin{abstract}
Background: Because of the previous high prevalence of resistance to usual antibiotics among uropathogens in Bangui, Central African Republic (CAR), a survey focused on Enterobacteriaceae, the most prevalent group responsible for urinary tract infections (UTIs), was conducted. The aim was to help health authorities revise antibiotic policies.

Methodology: We performed a retrospective analysis of all cases of confirmed UTIs due to Enterobacteriaceae in outpatients attending the Institut Pasteur de Bangui (IPB), CAR, between 2004 and 2006.

Results: During the study period, 560 (10.9\% of urine submissions) UTIs were confirmed and 443 Enterobacteriaceae strains were isolated, representing $79 \%$ of the causative agents for UTIs. Among these 560 strains, E. coli was the most common, representing $64 \%$ of the isolates, followed by $K$. pneumoniae (10\%) and other Enterobacteriaceae (5\%). Extended-spectrum beta-lactamase (ESBL) producing Enterobacteriaceae significantly increased from five (3.7\%) to thirty-three (19.3\%) between 2004 and 2006. A significantly increased resistance rate to nalidixic acid, ciprofloxacin and gentamicin was observed in ESBL-nonproducing Enterobacteriaceae over the study period.

Conclusions: Empiric treatment for UTIs in Bangui should be reconsidered and prudent use of antibiotics, particularly ciprofloxacin, is highly recommended. The recent spread of ESBL-producing Enterobacteriaceae in central African outpatients is striking and underlines the need for further studies.
\end{abstract}

Key words: urinary tract infection, extended-spectrum beta-lactamase, outpatients, Central Africa

J Infect Developing Countries 2009; 3(3):187-190.

Received 2 September 2008 - Accepted 12 January 2009

Copyright (C) 2009 Bercion et al. This is an open-access article distributed under the Creative Commons Attribution License, which permits unrestricted use, distribution, and reproduction in any medium, provided the original work is properly cited.

\section{Introduction}

Urinary tract infections (UTIs) are one of the most common infectious diseases diagnosed in outpatients and are frequently caused by Enterobacteriaceae strains. Most UTIs in developing countries are treated on an empirical basis; thus treatment should be based on available local data regarding the susceptibility of common pathogens to antibiotics. Unfortunately, most patients who can afford drugs are prone to self-treatment in the absence of any laboratory investigation. Therefore, an increase in antibiotic resistance is to be expected and it is important to determine the distribution of pathogens responsible for UTIs and their patterns of resistance to the main available antibiotics. In a previous survey, a high rate of resistance to commonly used antibiotics (amoxicillin, trimethoprim/sulfamethoxazole and quinolones) has already been described [1]. Such observations have also been made in other developing countries including Sudan [2], Madagascar [3], and Nicaragua
[4]. In contrast, lower resistance rates to these antibiotics have been reported in a recent study of pregnant women from Tanzania [5].

The Central African Republic (CAR) is one of the poorest countries in the world and generic drugs are often the only affordable antibiotics. This study aimed to assess the evolution of resistance of Enterobacteriaceae strains isolated from UTIs in Bangui to the antibiotics available in the country and, consequently, to aid health authorities in revising the recommended first-line antibiotic treatments.

\section{Materials and Methods}

We conducted a retrospective study of all nonduplicate Enterobacteriaceae strains isolated from outpatients with suspected uncomplicated UTIs who attended the Institut Pasteur de Bangui (IPB) between January 2004 and December 2006. Urine samples were cultured on bromocresol purple agar plates (bioMérieux, Marcy l'Etoile, France). Only urine from patients with pyuria ( $>10$ white blood cells $/ \mu \mathrm{L}$ ) 
Table 1. Evolution of the resistance of Enterobacteriaceae isolated from UTIs in Bangui between 2004 and 2006.

Percentage of isolates resistant to antibiotics (\%)

All Enterobacteriaceae

ESBL-nonproducing strains

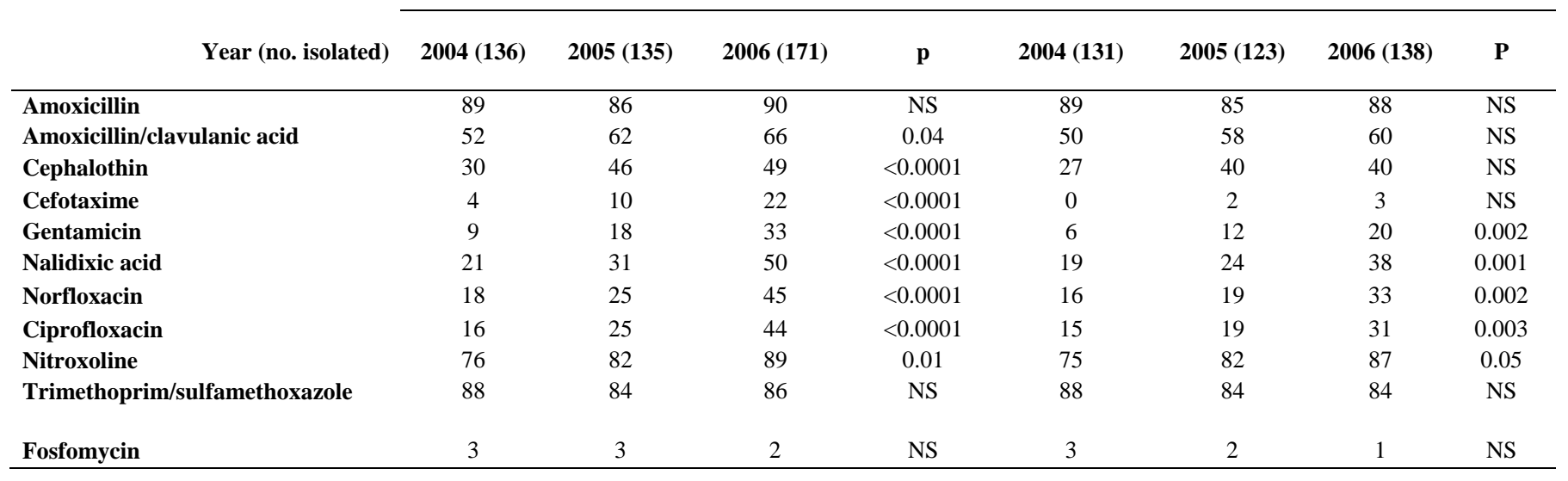

NS: non significant

and substantial bacteriuria ( $>1 \times 10^{5} \mathrm{cfu} / \mathrm{mL}$ ) involving a single pathogen was included. Enterobacteriaceae strains were Gram stained and then identified with API 20E strips (bioMérieux). Susceptibility of these strains to antibiotics was determined by the disc diffusion method on MuellerHinton agar as recommended by the Comité de l'antibiogramme de la Société Française de Microbiologie (CA-SFM; Antibiogram Committee of the French Microbiology Society) [6]. The following antibiotics were tested: amoxicillin, amoxicillin/clavulanic acid, ticarcillin, cephalothin, cefoxitin, cefotaxime, ceftazidime, gentamicin, nalidixic acid, norfloxacin, ciprofloxacin, trimethoprim-sulfamethoxazole, nitroxoline, and fosfomycin. Extended-spectrum beta-lactamase (ESBL) strains were systematically searched for by a double-diffusion method with cefotaxime or ceftazidime and amoxicillin/clavulanic acid as recommended [6]. Diameters of growth inhibition areas were measured with the automated Osiris system (Bio-Rad, Marnes la Coquette, France). Enterobacteriaceae strains were classified as susceptible, intermediate, or resistant according to the CA-SFM criteria. Intermediate and resistant strains were then grouped together and reclassified as resistant. Escherichia coli ATCC 25922 was used as a control strain.

To prove the increase in resistance of isolates with time, differences between proportions were tested using a $\mathrm{Chi}^{2}$ for trends (STATA version 8.0; Stat corp., Texas) A $P$-value of $<0.05$ was considered significant.

\section{Results}

From 2004 to 2006, bacterial pathogens were isolated from 560 of the $5,128(10.9 \%)$ patients who submitted urine samples at the IPB. Enterobacteriaceae strains were cultured from 443 (79\%) at a significant concentration, confirming UTIs: 357 (64\%) Escherichia coli, 57 (10\%) Klebsiella pneumoniae, 12 (2\%) Salmonella spp. and 17 (3\%) belong to other species (ten Proteus spp., three Enterobacter spp., four Citrobacter spp., one Morganella morganii). In 2004, 136 strains were isolated, 135 in 2005 and 171 in 2006. The distribution of strains among species did not vary significantly during this three-year period.

Among patients, the sex ratio was 1.5 with 267 female $(60 \%)$ and 176 male (40\%). Twenty-four percent of these 443 patients were under $15,49 \%$ in the age group 15 to 45 and $27 \%$ over 45 . There was also no significant difference in the distribution of species between age groups and genders.

Fifty ESBL-producing strains were identified in three years: 29 E. coli, $17 \mathrm{~K}$. pneumoniae, 3 Enterobacter spp. and one $M$. morganii. An increasing prevalence of ESBL-producing Enterobacteriaceae in UTIs was noteworthy: five (3.7\%) were detected in 2004, 12 (8.9\%) in 2005 and $33(19.3 \%)$ in 2006 ( $\mathrm{p}<0.001)$. These strains were as common in patients under 15 years old (22 strains) as in patients from 15 to 45 years old (19 strains).

Resistance to amoxicillin and trimethoprim/sulfamethoxazole was very high ( > $85 \%$ ) and stable over the three-year period. In contrast, only one to three percent of these strains 
were resistant to fosfomycin. Resistance to gentamicin (9 to 33\%), nalidixic acid (21 to 50\%), ciprofloxacin (16 to $44 \%$ ) and cefotaxime (4 to $22 \%$ ) increased significantly between 2004 and 2006 (Table 1). These data directly relate to the significant increase in ESBL-producing strains. In addition to high-level resistance to beta-lactams, 46 of 50 ESBLproducing Enterobacteriaceae were also resistant to ciprofloxacin. Additionally, resistance to gentamicin, quinolones, and fluoroquinolone of ESBLnonproducing strains also increased during this threeyear period.

\section{Discussion}

Urinary tract infections are a common problem in general practice and are usually treated empirically. Empirical treatments should be based on local data regarding common pathogens and their susceptibility to available antibiotics. Patients attending the IPB are likely a selected population with more complicated clinical courses or underlying debilitating conditions and thus may be more likely to harbour a resistant bacterium. Difficulties in obtaining representative data about distribution and susceptibility patterns of uropathogens from laboratory data are well-documented [7]. Our study population presenting with UTI was selected mainly because it could afford the cost of urine examination As the collection of data and the laboratory methods were consistent throughout the study period, the evolution of the susceptibility over this three-year period should represent the general trend among urinary tract pathogens.

As reported previously in Bangui [1] and in other developing $[2,8]$ as well as in developed countries [9$11], E$. coli was the main pathogen responsible for UTIs, followed by $K$. pneumoniae.

According to multicentre studies conducted in Europe [9,11] and in North America [10], resistance to amoxicillin and trimethoprim/sulfametoxazole in UTIs from E. coli is no higher than $38 \%$ and $30 \%$, respectively; however, there were important differences between neighbouring countries. A very high rate of resistance to these two antibiotics is observed in Bangui that can be explained by the high selective pressure exerted by an intensive use of inexpensive and easily administered drugs for many years; moreover, trimethoprim/sulfamethoxazole is commonly used to prevent HIV-associated opportunistic infections. Finally, the distribution of antimicrobial agents in pharmacies in the absence of any medical prescription and the sale of inexpensive drugs of uncertain origin in the parallel drug markets may contribute to these high resistance rates.

In this study, the increasing resistance of $E$. coli and $K$. pneumoniae to third-generation cephalosporin over three years is striking. This phenomenon is associated with the presence of ESBLs. The diffusion of a CTX-M-15 type beta-lactamase in clinical strains of $E$. coli from Bangui has recently been reported [12]. The number of bacteria presenting the same antibiogram profile as these ESBL-producing strains is increasing, even in patients with communityacquired infections who were observed from 2004 to 2006 [12]. This spread of ESBL-producing strains (particularly CTX-M enzymes) at the community level, causing disease or colonisation, has been previously reported [13]. It was not possible to identify risk factors associated with ESBL-producing strains because our study was retrospective; however, the very common use of generic ciprofloxacin for treatment of both in- and outpatients in Bangui may have contributed to a selection of these ESBLproducing strains, which are often multi-resistant, specifically to ciprofloxacin, trimethoprim/sulfamethoxazole and gentamicin. Thus, these strains are difficult to treat, particularly in resource-poor countries where the number of available drugs is limited.

Nevertheless, increasing resistance to fluoroquinolones and gentamicin are also observed for ESBL-nonproducing Enterobacteriaceae. For example, prevalence of resistance to ciprofloxacin, which was at $10 \%$ in 2003 [1], went up to $31 \%$ in 2006 (table 1).

Our findings emphasize the need to reconsider the general use of antibiotics in CAR and highlight the lack of information about antibiotic use and drug resistance among the medical population. We strongly advise the prudent use of ciprofloxacin to prevent not only a further increase of resistance, but also a similar rapid occurrence of a very high level of resistance that was observed with trimethoprim/sulfamethoxazole and amoxicillin. A large survey of ESBL-producing clinical isolates will be conducted in Bangui to determine the main risk factors of the acquisition of such strains and to facilitate the planning of preventive actions, thus preventing the spread of these threatening pathogens.

\section{Acknowledgements}

This work was financed by grants from the French Ministère des Affaires Etrangères, FSP 2001-168, “Appui 
au réseau de surveillance des résistances aux antimicrobiens."

\section{References}

1. Hima-Lerible H, Ménard D, Talarmin A (2003) Antimicrobial resistance among uropathogens that cause communityacquired urinary tract infections in Bangui, Central African Republic. J Antimicrob Chemother 51: 192-194.

2. Ahmed AA, Osman H, Mansour AM, Musa HA, Ahmed AB, Karrar Z, Hassan HS (2000) Antimicrobial agent resistance in bacterial isolates from patients with diarrhoea and urinary tract infection in the Sudan. Am J Trop Med Hyg 63: 259263.

3. Randrianirina F, Soares JL, Carod JF, Ratsima E, Thonnier V, Combe P, Grosjean P, Talarmin A (2006) Antimicrobial resistance among uropathogens that cause communityacquired urinary tract infections in Antananarivo, Madagascar. J Antimicrob Chemother 59: 309-312.

4. Matute AJ, Hak E, Schurink CA, McArthur A, Alonso E, Paniagua M, Van Asbeck E, Roskott AM, Froeling F, Rozenberg-Arska M, Hoepelman IM (2004) Resistance of uropathogens in symptomatic urinary tract infections in León, Nicaragua. Int J Antimicrob Agents 23: 506-509.

5. Blomberg B, Olsen BE, Hinderaker SG, Langeland $\mathrm{N}$, Gasheka P, Jureen R, Kvale G, Midtvedt T (2005) Antimicrobial resistance in urinary bacterial isolates from pregnant women in rural Tanzania: implications for public health. Scand J Infect Dis 37: 262-268.

6. Comité de l'Antibiogramme de la Société Française de Microbiologie (CA-SFM). Communiqué 2005, Paris, France: http://www.sfm.asso.fr.

7. Ti TY, Kumarasinghe G, Taylor MB Tan SL, Ee A, Chua C, Low A (2003) What is true community-acquired urinary tract infection? Comparison of pathogens identified in urine from routine outpatient specimens and from community clinics in a prospective study. Eur J Clin Microbiol Infect Dis 22: 242245.

8. Dromigny JA, Ndoye B, Macondo EA, Nabeth P, Siby T, Perrier-Gros-Claude JD (2003). Increasing prevalence of antimicrobial resistance among Enterobacteriaceae uropathogens in Dakar, Senegal: a multicenter study. Diagn Microbiol Infect Dis 47: 595-600.

9. Kahlmeter G (2003) An international survey of the antimicrobial susceptibility of pathogens from uncomplicated urinary tract infections: the ECO-SENS Project. J Antimicrob Chemother 51: 69-76.

10. Zhanel GG, Hisanaga TL, Laing NM, De Corby MR, Nichol KA, Weshnoweski B, Johnson J, Noreddin A, Low DE, Karlowsky JA; for the NAUTICA group, Hoban DJ (2006) Antibiotic resistance in Escherichia coli outpatient urinary isolates: final results from the North American Urinary Tract Infection Collaborative Alliance (NAUTICA). Int $\mathrm{J}$ Antimicrob Agents 27: 468-475.

11. Goldstein FW and the Multicentre Study Group (2000) Antibiotic susceptibility of bacterial strains isolated from patients with community-acquired urinary tract infections in France. Eur J Clin Microbiol Infect Dis 19: 112-117.

12. Frank T, Arlet G, Gautier V, Talarmin A, Bercion R (2006) Extended-spectrum beta-lactamase-producing Enterobacteriaceae, Central African Republic. Emerg Infect Dis 12: 863-865.

13. Calbo E, Romani V, Xercavins M, Gomez L, Vidal CG, Quintana S, Vila J, Garau J (2006) Risk factors for community-onset urinary tract infections due to Escherichia coli harbouring extended-spectrum $\beta$-lactamases. J Antimicrob Chemother 57: 780-783.

\section{Corresponding author}

R. Bercion, Hôpital Principal de Dakar, Avenue Nelson Mandela, PO BOX 3006, Dakar, Senegal.

Phone (221) 338395810

Fax (221) 338395088

Email: rbercion@hotmail.com

Conflict of interest: No conflict of interest is declared. 